Research Article

\title{
Scientific and Practical Justification of the Parameters of the Process Chambers with Superhigh Frequency Power Supply
}

Chuvash State Agricultural Academy, K. Marx St., 29, Cheboksary, Chuvash Republic, Russian Federation, 428003

Received 4 July 2016; Accepted 18 September 2016

\begin{abstract}
According to statistical data of the Russian Federation, the volume of production of fermented milk products is growing. Up to $20 \%$ of the total volume of produced fermented milk products is processed to obtain a variety of different products. In this case, specific power consumption for processing of fermented milk products remains quite high, and the quality of products is not always satisfactory. Therefore, the development of resource-saving technology of heat treatment of fermented milk products and the related plants is a relevant task. We have justified a new method of processing of fermented milk products, implemented in superhigh frequency-induction heat treatment plant for curdy raw materials and in superhigh frequency heat treatment plant for butter.
\end{abstract}

Keywords: Electrodynamic system, waveguide, electromagnetic field.

\section{Introduction.}

Technical means used for heat treatment of food raw materials with conventional electric heaters are quite energyintensive, and the shelf life of manufactured products is short. The use of conveyor superhigh frequency plants will improve technical and economic performance of the production process, which is made possible by the use of the evanescent-mode waveguide providing absorption of superhigh frequency energy that seeps through the slot, providing transportation of raw materials.

The analysis of methods of food product heat treatment (convective, conductive and dielectric) leads to the conclusion about the possibility of improving the quality of products as well as reducing power consumption through the use of the electromagnetic field [8].

\section{Method.}

To ensure the continuous process of food raw material heat treatment in superhigh frequency electromagnetic field, superhigh frequency waveguide and resonator plants with complex linking comb-type devices are used.

In order to generate endogenous heat in food raw material in the continuous mode, and to limit the radiation of superhigh frequency energy from the resonator to the environment through the gap, the evanescent-mode waveguide in the form of a cylindrical segment is attached to the generator. In connection with this, a new method of heat treatment of food raw materials is developed, implemented in superhigh frequency-induction plant [9].

\footnotetext{
*E-mail address: editorl@ucademicpapers.org

ISSN: $1791-2377$ @ 2016 Eastern Macedonia and Thrace Institute of Technology. All rights reserved.
}

\section{Main part.}

In order to generate endogenous heat in the processed food raw materials in the continuous mode, we have developed two types of electrodynamic systems that are part of the developed superhigh frequency-induction plant for heat treatment of fermented milk products and superhigh frequency butter melter, respectively.

The first type of electrodynamic system is designed for superhigh frequency plants where single-piece food raw materials are heat-treated. It provides limitation of leakage of superhigh frequency energy radiation from the cavity through the slot. The evanescent-mode waveguide is attached to the chamber in the form of a cylindrical segment; the waveguide is diaphragmed. As a result, an innovative method of heat treatment of food raw materials in superhigh frequency-induction plant is justified.

The technical result of heat treatment process is to accelerate the technology, as well as to improve the quality of finished products. These results are achieved through the multiple combined exogenous and endogenous heating in superhigh frequency-induction plant comprising a process chamber of the resonator cavities with diaphragmed waveguides located outside of the resonator, and induction heaters.

The developed electrodynamic system includes: a resonator cavity, a cathode, an anode block, devices to output superhigh frequency energy and a magnetic system. The system provides power needed for the production process, and compliance with the temperature modes in the resonator. The system comprises input and output devices, which eliminate leakage of electromagnetic radiation when opening the process chamber for product loading and unloading. Special resonator cavities, various waveguides and slowing systems are used for this purpose. In the development of conveyor superhigh frequency plants, main difficulties arise in the design of loading devices, as strict 
requirements apply to the power of radiated electromagnetic flux into the environment.

The main objective in the development of superhigh frequency plants is the design of the process chamber, providing transformation of the superhigh frequency field energy to the heat energy in the product subjected to heat treatment. Sizes of superhigh frequency resonator cavities used in these plants, are several times higher than the wavelength. Also, the emergence of several crests of the standing wave and nodes in the resonator is the cause of uneven heating.

Dielectric heating in food production provides a high power at lowered parameters of the electric field. In heat treatment, raw material is subjected to a standing electromagnetic wave. It occurs in the resonator cavities. In the waveguides, food raw material is subjected to the running electromagnetic wave, it is reasonable to use in superhigh frequency plants of continuous action. The electromagnetic wave within the waveguide of rectangular shape propagates provided that the size of the wall with the greatest width is greater than $1 / 2$ of the radiated wavelength [6]. In case where the electromagnetic wave length is 2 times greater than the size of the wide wall of the waveguide, the wave is damped according to the aperiodic law. If these parameters are equal, it will correspond to the lowest critical frequency of the waveguide - for a wave of $\mathrm{TE}_{10}$ type. When selecting the size of the waveguide cross section, such wave attenuation can be achieved that the gap for transportation of raw material will not result in distortion of the electromagnetic field structure, and radiation from the waveguide surface can be prevented.

Energy reflection from the edge of the waveguide is unacceptable because it would lead to formation of a standing wave, and it violates the uniformity of food raw material heating. This would also violate the operation of superhigh frequency generators and may cause overheating of the magnetrons, and thus their failure.

The plant that we have developed is a conveyor system with generators and evanescent-mode diaphragmed waveguides. Energy from the generator is directed to the product heating zone.

Devices for loading and unloading of containers with raw material should ensure service personnel safety and efficient operation of the plant developed. Evanescent-mode waveguides (attenuators) are used in the proposed superhigh frequency plant. The waveguides represent a diaphragmed segment of cylindrical shape.

Sizes of the walls of the waveguide cross section are less than half a wavelength.

There are systems to slow down the power of electromagnetic radiation flux, where the length of the electromagnetic wave is less than the length of a wave in space. A slowing system is developed as a cylindrical waveguide diaphragmed throughout the entire volume. For each geometry of the evanescent-mode waveguide there is a certain frequency range where the interaction efficiency is maximized.

It was found that the wave gains maximum power in the center of the resonator cavity, where the electric component of the electromagnetic field reaches maximum values. Slots can be made in the walls of the resonator chamber, however to prevent radiation, certain rules must be followed. A slot in the wall must provide continuity of the lines of currents that flow along the walls of the resonator cavity. The currents in rectangular resonator are directed along the wide walls and across the narrow walls. Therefore, the slots in the walls may be located anywhere. When designing the slots, the value of gaps above and below the conveyor with raw material was summed with the thickness of a metallized dielectric, as well as with the depth of containers with raw material.

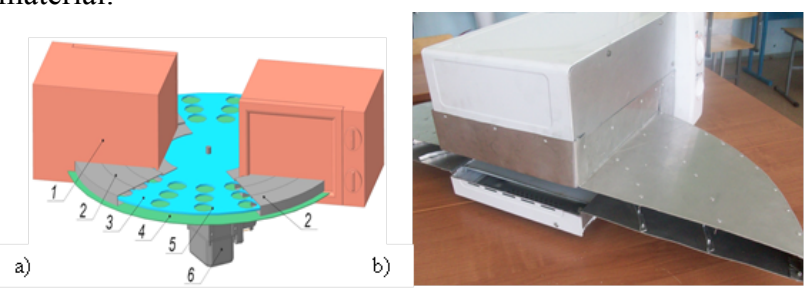

Fig. 1. Resonator chamber with diaphragmed waveguide attached: a) 3D model; b) actual chamber

Because of the gap in the resonator chamber of superhigh frequency-induction plant, the power of electromagnetic radiation flux is propagated. To prevent propagation of radiation, a waveguide must be provided. Waves propagate along the waveguide at the speed several times less than the speed of light. The waveguides have several advantages: simplicity and rigidity of the structure, the electromagnetic field is confined within, and thus there are no energy losses. When the waveguide is filled with air, losses in the dielectric material are small, and the dielectric strength is greater. In superhigh frequency range, wave attenuation in the waveguide is $0.01 \mathrm{~dB}$ per $1 \mathrm{~m}$ of length. The disadvantage of the waveguide is the need to take into account the dependence of wave propagation speed on its frequency, so we must calculate certain sizes of crosssection for each type of the waveguide. There is a certain relationship between the length of the electromagnetic wave in the waveguide, wavelength in the space and the critical length of the electromagnetic wave. In case where the ratio of the wavelength to the critical parameters of the length is greater than one, this type of the electromagnetic wave cannot propagate, and "evanescent-mode" waveguide is used for this type of wave. Evanescent-mode waveguides in the form of metal tubes, where the wave cannot propagate, are more known.

When the waveguide is filled with raw material, we can increase the critical wavelength, and in this case it is not necessary to change the dimensions of the waveguide. The main goal is to achieve energy leakage of the electromagnetic radiation not exceeding 2 to $3 \mu \mathrm{W} / \mathrm{cm}^{2}$ at a distance of $15 \mathrm{~cm}$ from the waveguide. These values are the lowest in the world and are completely safe for the personnel.

The developed evanescent-mode waveguide provides an exponential reduction in power along the diaphragm.

Due to fringing flux of electromagnetic radiation power, food raw material maintains temperature mode of the heater outside the resonator chamber as well [5].

The increment of the temperature of food raw material inside the resonator chamber (up to $28 \mathrm{~cm}$ ) is between 68 and $48{ }^{\circ} \mathrm{C}$, and $3{ }^{\circ} \mathrm{C}$ outside the chamber at a distance of 40 $\mathrm{cm}$ from the source, and $2^{\circ} \mathrm{C}$ at a distance of $50 \mathrm{~cm}$.

Justification of the nodes of superhigh frequency generator is in definition of the quality factor and capacity, as well as in the calculation of the electric field intensity. Evanescent-mode diaphragmed waveguide is justified, differing from the comb-like slowing system by the fact that at an equal step, diaphragm radius location changes. Also we have studied the distribution pattern of the electric field and determined slowness factor as a function of diaphragm 
diameter. We have justified the size of the slot in the resonator chamber and the configuration of evanescentmode diaphragmed waveguide. The size of the slot in the resonator is matched to the dimensions of containers used, selected in accordance with the calculated depth of penetration of the electromagnetic field in raw material.

The waveguide is diaphragmed to slow down the wave, as well as to increase performance of superhigh frequency plant, since in its absence, the plant overheats the magnetron of the second generator fails. We have determined the quality factor of the designed resonator chamber in case it has a slot for movement of the disk conveyor and the presence of evanescent-mode diaphragmed waveguide.

In design of a resonator cavity, we must estimate its quality factor $[6,7,11]$ :

$$
Q=\frac{V}{\Delta V \cdot \operatorname{tg} \delta},
$$

where $\mathrm{V}$ - the volume of the designed resonator; $\Delta \mathrm{V}$ - the volume of load.

$$
Q=\frac{20}{0,3 \cdot 0,2}=333 .
$$

The quality factor of the designed process chamber of superhigh frequency-induction plant under normal load with food product reaches a few hundred units.

The effect of the volumetric heating of the product when it is heat-treated in the electromagnetic field of superhigh frequency range is achieved due to field penetration into the product to a certain depth. At a known depth of penetration of the electromagnetic field, it is possible to calculate the shape and volume of containers for food raw material, taking into account the requirements of the technology and parameters of the electromagnetic field. Heat treatment of raw materials must be conducted in dielectric containers because they are not heated in the electromagnetic field of superhigh frequency (silicone containers are suitable). Product dimensions should not exceed 2-3 values of the electromagnetic field penetration depth. On the basis of calculations made it is found that optimal containers are in the form of a low cylinder, with a height $2 \ldots 5$ times less than their diameter.

The depth of penetration of the electromagnetic field in food raw material means the distance from the surface of the product inside, along which the power capacity of internal heat sources decreases 2.73 times. The power flow is directed from the surface of the product inside and attenuates exponentially with its distribution in raw material. As a result of the study of the depth of penetration of the electromagnetic field of superhigh frequency in food raw material, we have determined the dimensions and shape of the product being processed in the chamber of superhigh frequency-induction plant. From the equations for electromagnetic wave propagation constant it is known that the penetration depth is equal to:

$$
\Delta=9.55 \cdot 10^{9} /(f \cdot \sqrt{\varepsilon} \cdot \operatorname{tg} \delta) \mathrm{cm},
$$

where $f$ - the frequency of the electromagnetic field in $\mathrm{Hz} ; \varepsilon$ - the dielectric constant of food raw material (36.6...47); $\operatorname{tg} \delta$ - the dielectric loss tangent of food (curdy) raw material equal to $0.158 \ldots 0,282 ; \varepsilon_{\mathrm{o}}=8.85 \cdot 10^{-12}$

$\mathrm{F} / \mathrm{m}$ - the absolute permittivity of vacuum.

The depth of penetration of superhigh frequency electromagnetic field in the product is:

$\Delta=9.55 \cdot 10^{9} /\left(2450 \cdot 10^{6} \cdot \sqrt{36.6 \ldots 47} \cdot(0.158 \ldots 0.282)=\right.$ $(4.29 \ldots 2.7) \mathrm{cm}$.

Calculations of the depth of penetration of the electromagnetic field in the product show that the uniform flow distribution by volume of raw material is possible, subject to certain dimensions - they should not exceed $2 \cdot(2.7 \ldots 4.29)=5.4 \ldots 8.58 \mathrm{~cm}$. Given the depth of penetration of the electromagnetic field, the dimensions of the silicone container must not exceed the calculated values. Heat treatment of raw material is offered to be conducted in the silicone containers of cylindrical shape. Containers of this shape are commercially available and have the following parameters: diameter $-7 \mathrm{~cm}$, depth $-2 \mathrm{~cm}$, volume $-77 \mathrm{~cm}^{3}$.

The second type of the electrodynamic system is intended for heat treatment of viscous food raw materials, namely butter.

The design parameters of the resonator chambers must provide uniform heating of raw material in the chamber throughout the entire volume. The resonator chambers must also have large throughput and fully use the power of superhigh frequency generator. Taking into account these requirements, we recommend using perforated resonator chambers for heat treatment of viscous food raw material. The chambers are constructed in the form of cylindrical resonator cavities that provide continuous process of raw material heat treatment.

When using superhigh frequency, a resonator cavity is used as a device with a resonance circuit with high quality factor. The resonator is a hollow cylinder, its surface is perforated, it is made of aluminum. The resonator diameter is designed taking into account the length of the electromagnetic wave which is $12.24 \mathrm{~cm}$. In the resonator shaped as a cylinder, electromagnetic field energy is distributed throughout the resonator cavity [1].

Let's define the types of waves generated in a rectangular resonator cavity with transverse dimensions of $12.24 \times 12.24 \mathrm{~cm}$ and in a cylindrical resonator cavity with a diameter of $12.24 \mathrm{~cm}$ at a frequency of electromagnetic field of superhigh frequency of $2450 \mathrm{MHz}$. The critical length of the electromagnetic wave depends on transverse dimensions of resonator $\mathrm{a}$ and $\mathrm{b}$ :

$$
\lambda_{c r}=\frac{1}{\sqrt{(m / 2 a)^{2}+(n / 2 b)^{2}}}, \mathrm{~cm},
$$

where $\mathrm{m}$ and $\mathrm{n}-$ the integer numbers.

Table 1 lists $\lambda_{c r}$ values for different $\mathrm{m}$ and $\mathrm{n}$ values

1) at a $=\mathrm{b}=12.24 \mathrm{~cm}$;

2) at $\mathrm{a}=\mathrm{b}=16 \mathrm{~cm}$.

The chamber diameter:

1) $16 \mathrm{~cm}$,

2) $12.24 \mathrm{~cm}$,

The length of both chambers is $12.24 \mathrm{~cm}$. 
Table 1. The values of critical length of the electromagnetic wave for different $\mathrm{m}$ and $\mathrm{n}$

\begin{tabular}{|c|c|c|c|c|c|c|c|}
\hline \multicolumn{8}{|c|}{ 1) at $\mathrm{a}=\mathrm{b}=12.24 \mathrm{~cm}$} \\
\hline $\mathrm{m}$ & 0 & 0 & 1 & 1 & 1 & 0 & 1 \\
\hline $\mathrm{n}$ & 1 & 2 & 0 & 1 & 2 & 3 & 3 \\
\hline \multirow{2}{*}{$\lambda_{c r}$} & 24.48 & 12.2 & 24.4 & 17.3 & 10.9 & 8.1 & 7.7 \\
\hline & & 4 & 8 & 1 & 48 & 6 & 413 \\
\hline $\mathrm{m}$ & 0 & 2 & 2 & 2 & 1 & 0 & 3 \\
\hline $\mathrm{n}$ & 4 & 0 & 1 & 2 & 4 & 5 & 6 \\
\hline \multirow{2}{*}{$\lambda_{c r}$} & 6.12 & 12.2 & 10.9 & 8.65 & 5.93 & 4.8 & 3.6 \\
\hline & & 4 & 48 & 5 & 73 & 96 & 493 \\
\hline \multicolumn{8}{|c|}{ 2) at $a=b=16 \mathrm{~cm}$} \\
\hline $\mathrm{m}$ & 0 & 0 & 1 & 1 & 1 & 0 & 1 \\
\hline $\mathrm{n}$ & 1 & 2 & 0 & 1 & 2 & 3 & 3 \\
\hline \multirow{2}{*}{$\lambda_{c r}$} & 32 & 16 & 32 & 22.6 & 14.3 & 10.6 & 10. \\
\hline & & & & 27 & 11 & 67 & 119 \\
\hline $\mathrm{m}$ & 0 & 2 & 2 & 2 & 1 & 0 & 3 \\
\hline $\mathrm{n}$ & 4 & 0 & 1 & 2 & 4 & 5 & 6 \\
\hline \multirow[t]{2}{*}{$\lambda_{c r}$} & 8 & 16 & 14.3 & 11.3 & 7.76 & 6.4 & 4.7 \\
\hline & & & 11 & 14 & 11 & & 703 \\
\hline
\end{tabular}

The table shows that at a frequency of $2450 \mathrm{MHz}(12.24$ $\mathrm{cm}), \mathrm{TE}_{\mathrm{mn}}$ wave can propagate in the resonator chamber if a $=\mathrm{b}=12.24 \mathrm{~cm}$ at:

1) $\mathrm{m}=0$ and $\mathrm{n}=1$;

2) $m=0$ and $n=2$;

3) $m=1$ and $n=0$;

4) $m=1$ and $n=1$.

if $\mathrm{a}=\mathrm{b}=16 \mathrm{~cm}$ at:

1) $\mathrm{m}=0$ and $\mathrm{n}=1$;

2) $m=0$ and $n=2$;

3) $m=1$ and $n=0$;

4) $m=1$ and $n=1$;

5) $m=1$ and $n=2$;

6) $m=2$ and $n=0$;

7) $m=2$ and $n=1$.

We have calculated the size of the perforations and the frequency of their arrangement on the chamber surface, given the multiplicity of one-fourth of the wavelength (to limit the flow of radiated power of the electromagnetic radiation through the perforations). We have also calculated the chambers throughput at different sizes of the particle passing through the raw material chamber [3].

Alignment of constructional parameters and modes of operation of superhigh frequency butter melter is performed on the basis of the available equations and taking into account the shape of (cylindrical) perforated resonator chamber, as well as the changes in the dielectric properties of the butter during its heat treatment.

Based on the methodology of V.I. Fedyanovich [10], we have analyzed the wave field, which propagates along the perforations of the resonator chamber.
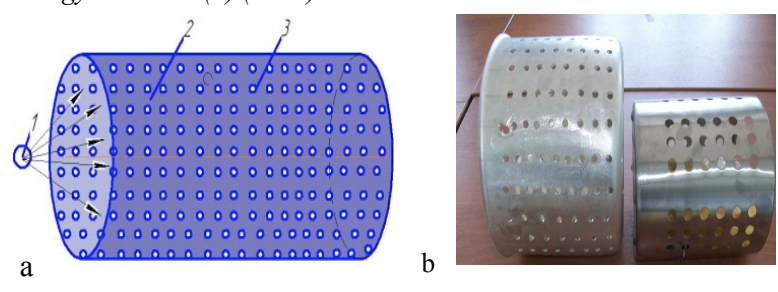

Fig. 2. Perforated resonator chamber: a) 3-D model; b) actual chamber of different capacity

For the perforated plane with the use of time dependence $\exp (\mathrm{i} \omega \mathrm{t})$, it is possible to express the field parameters through inductance of the perforations $\mathrm{L}$ and their capacity C:

$$
\begin{aligned}
& E_{\mathrm{t}} \cdot \mathrm{i} \cdot \omega \cdot C_{\mathrm{t}}=H_{\mathrm{s}}^{+}-H_{\mathrm{s}}^{-}+\mu_{0} \frac{\mathrm{d}}{\mathrm{ds}}\left(\mathrm{H}_{\mathrm{n}} / \mathrm{L}_{\mathrm{n}}\right), \\
& \mathrm{E}_{s}=\mathrm{i} \cdot \omega \cdot \mathrm{L}_{\mathrm{t}}\left(\mathrm{H}_{\mathrm{t}}^{-}-\mathrm{H}_{\mathrm{t}}^{+}\right)+\varepsilon_{0} \frac{d}{d \mathrm{~s}}\left(\left(E_{\mathrm{t}}^{-}-E_{\mathrm{t}}^{+}\right) / C_{\mathrm{n}}\right),
\end{aligned}
$$

Where index $(+)$ refers to the resonator surface $n=-0$,

index $(-)$ - to the surface $n=+0 ; t, s$,

$\mathrm{n}$ - directions,

$\mathrm{t}$ - a coordinate in the transverse plane of the resonator chamber,

$\mathrm{s}$ - along the plane,

$\mathrm{n}$ - normal to the plane;

$\mu_{0}$ - magnetic permeability of vacuum, $\mathrm{H} / \mathrm{m}$;

$\varepsilon_{0}-$ permittivity of vacuum, $\mathrm{F} / \mathrm{m}$;

$\mathrm{E}, \mathrm{H}-$ the values of intensity of electric and magnetic component of the field.

The maximum level of the electric field intensity and the transverse component of the electromagnetic field in the developed perforated resonator chamber are offset by one fourth of the wavelength. The index at capacity $\mathrm{C}$ indicates the direction of the electric field (near the chamber surface at the localization point), and the index at inductance $\mathrm{L}$ indicates the orientation of the magnetic field. By solving these equations we find:

$$
\begin{aligned}
& \mathrm{L}_{\mathrm{n}}=\pi \cdot \mu_{0} /\left[2 T \cdot \ln \left(1 / \cos \left(\mathrm{q}^{\prime} / 2\right)\right)\right], \\
& C_{\mathrm{t}}=\left(2 \varepsilon_{0} / \pi\right) / T \cdot \ln \left(1 / \cos \left(\mathrm{q}^{\prime} / 2\right)\right), \\
& \mathrm{L}_{\mathrm{t}}=\mu_{0} \cdot T \cdot \ln \left(1 / \cos \left(\mathrm{p}^{\prime} / 2\right)\right) / 2 \pi, \\
& C_{\mathrm{n}}=2 \pi \cdot \varepsilon_{0} / t \cdot \ln \left(1 / \cos \left(\mathrm{p}^{\prime} / 2\right)\right),
\end{aligned}
$$

where $\mathrm{T}-$ the period of perforation alternation,

$$
\mathrm{q}^{\prime}=\pi \cdot \mathrm{q} / \mathrm{T},
$$

$\mathrm{q}$ - the width between the perforations on the resonator surface; $p^{\prime}=\pi \cdot p / \mathrm{T}$,

$$
\begin{aligned}
& \mathrm{p}-\text { the perforation diameter, } \\
& \mathrm{C}_{\mathrm{t}} / \mathrm{T} \text { - capacity per unit of length, } \\
& \mathrm{L}_{\Pi} \cdot \mathrm{T} \text { - inductance per unit length. }
\end{aligned}
$$

Let's assume that $\mathrm{H}_{\mathrm{s}}=0$, and that the electromagnetic field propagates along the perforations made (along s). Let's specify the voltage at the perforation as $U=E_{t} \cdot T$ and the current at its edges as 
$\mathrm{I}=-\mathrm{B}_{\mathrm{n}} / \mathrm{L}_{\mathrm{n}}$,

where $\mathrm{B}_{\mathrm{n}}=\mu_{\mathrm{o}} \cdot \mathrm{H}_{\mathrm{n}}$ - the magnetic induction

From Maxwell's equations we find $\mathrm{dU} / \mathrm{ds}=\mathrm{i} \cdot \omega \cdot \mathrm{B}_{\mathrm{n}} \cdot \mathrm{T}$

And obtain the following equations:

$\mathrm{i} \cdot \omega \cdot\left(\mathrm{C}_{\mathrm{t}} / T\right) \cdot \mathrm{U}+\mathrm{dI} / \mathrm{ds}=0$,

$\mathrm{i} \cdot \omega \cdot\left(\mathrm{L}_{\mathrm{n}} \cdot T\right) \cdot I+\mathrm{dU} / \mathrm{ds}=0$.

Perforation of the chamber is partially filled with a dielectric (butter) so that the boundary of the product coincides with an equipotential surface of the chamber. In this case, we can determine capacity $C_{n}$.

Let's determine capacities for different schemes of filling of the developed perforated resonator chamber:

a) $\mathrm{C}_{\Pi(\mathrm{v})}=\mathrm{C}_{\Pi} \cdot(1-\mathrm{V}+\mathrm{V} / \varepsilon)$,

b) $\mathrm{C}_{\mathrm{n}(\mathrm{v})}=\mathrm{C}_{\Pi} \cdot(1-(1-1 / \varepsilon) \cdot \mathrm{V} / 2) /(1-(1-1 / \varepsilon) \mathrm{V})$,

c) $\mathrm{C}_{\mathrm{t}(\mathrm{v})}=\mathrm{C}_{\mathrm{t}} \cdot(1-\mathrm{V} \cdot \varepsilon-\mathrm{V})$,

d) $\mathrm{C}_{\mathrm{t}(\mathrm{v})}=\mathrm{C}_{\mathrm{t}} \cdot(1-\mathrm{V} \cdot(\varepsilon-1) / 2$,

where $\mathrm{V}$ - the filling factor of the perforations with a dielectric (butter).

In case of complete filling of the perforations with the product (butter), capacities increase $\varepsilon$ times. Knowing the capacity of the developed perforated resonator chamber, we can determine power losses during penetration into the product according to the well-known equation:

$\mathrm{P}=\mathrm{U}^{2} \cdot 2 \pi \cdot f \cdot \mathrm{C} \cdot \operatorname{tg} \delta$,

where $\operatorname{tg} \delta=0.0939 \cdot \mathrm{T}^{0,0894}-$ dielectric loss tangent of butter;

$\varepsilon=3.5331 \cdot \mathrm{T}^{0,0645}-$ dielectric permittivity of the product, changing during heat treatment at a frequency of $2450 \mathrm{MHz}$.

The definitions of the inductance and capacity of the perforations of the resonator chamber normal to the plane of the electric component, as well as the magnetic component of the electromagnetic field also in the transverse plane of the resonator chamber, allow us to calculate the quality factor and losses of EMI flow through the perforations of the chamber. It is known that the quality factor of the resonator chamber without perforations is expressed as follows:

$\mathrm{Q}_{2}=\omega_{\mathrm{o}} \cdot \mathrm{W}_{\mathrm{o}} / \mathrm{P}$,

where $\omega_{0}$ - the natural frequency of the resonator circuit, $1 / \mathrm{sec}$;

$\mathrm{W}_{\mathrm{o}}$ - the energy accumulated in the circuit, $\mathrm{W} \cdot \mathrm{h}$;

$\mathrm{P}$ - the power of absorption by raw material, $\mathrm{W}$.

The derived expressions of the inductance and capacity of the perforations of the perforated resonator chamber normal to the plane of the intensity of the electric and magnetic field component, as well as along the transverse plane of the resonator chamber, allow us to calculate the quality factor $\left(Q_{1}\right)$ and losses of EMI flow through the perforations of the chamber. The study of the quality factor of the resonator chamber without the perforations $\left(Q_{2}\right)$ and the quality factor of the perforations allows estimating the quality factor of the perforated resonator chamber [2]:

$Q_{1}=\frac{1}{R} \cdot \sqrt{\frac{L}{C}}, Q_{2}=\frac{\omega_{0} \cdot W_{0}}{P}$,

where $\omega_{0}$ - the natural frequency of the resonator circuit, $1 / \mathrm{sec}$;

$\mathrm{W}_{0}$ - the energy accumulated in the circuit, $\mathrm{W} \cdot \mathrm{h}$;

$\mathrm{P}$ - the power of absorption by raw material, $\mathrm{W}$.

It is known that in the resonator cavities as in the circuit with lumped parameters $\mathrm{L}, \mathrm{C}$, oscillation process is in continuous conversion of electric field energy into magnetic field energy and then back in a quarter of a period. Therefore, they are evaluated the same way - by the internal quality factor, by expressing it through the reactive energy accumulated at resonance $\mathrm{W}_{\mathrm{x}}=\mathrm{L} \cdot \mathrm{I}_{\mathrm{m}}^{2} / 2$, and energy losses during one period of oscillations

$\mathrm{W}_{\mathrm{r}}=\mathrm{T}_{0} \cdot \mathrm{I}_{\mathrm{m}}^{2} \cdot \mathrm{R} / 2$,

$\mathrm{Q}=\omega_{0} \cdot \mathrm{L} / \mathrm{R}=2 \pi \cdot \mathrm{W}_{\mathrm{x}} / \mathrm{W}_{\mathrm{r}}$,

where $I_{m}$ - the amplitude of the current in the circuit,

$\mathrm{R}$ - the active resistance.

Large volume of the resonator allows it to accumulate much more energy $W_{x}$, and the absence of dielectric losses and radiation losses, as well as small losses due to conduction currents contribute to much less loss energy $\mathrm{W}_{\mathrm{r}}$, than in LC circuit. Thus, the internal quality factor of resonator cavities for $\mathrm{H}_{011}$ wave types reaches tens of thousands in a cylindrical resonator.

Heat loss in the designed resonator cavity is rather great due to its perforation, since perforation violates screening of the flow of the electromagnetic radiation. However, the great advantage of the perforated resonator chamber is that the loss of flow of the electromagnetic radiation through it is effectively used by raw material located on the surface of the chamber, and namely due to the emerging edge effect of the flow of the electromagnetic radiation, food raw material is preheated, and this affects maintenance of the efficiency of superhigh frequency generator. It is possible to reduce the intensity of the flow of the electromagnetic radiation through the perforations in the chamber if the perforation radius is $10 \ldots 15$ times smaller than the operating wavelength. It means that at the electromagnetic wavelength equal to 12.24 $\mathrm{cm}$, the optimum radius of the perforations of the resonator chamber is from 0.8 to $1.2 \mathrm{~cm}$. The radius of the perforations of the developed and designed resonator chambers is 0.25 $\mathrm{cm}$ and $0.35 \mathrm{~cm}$. It is 4 times less than the maximum permissible parameters. In this case, attenuation of the power flow of the electromagnetic radiation is equal to 45.7 - $64 \mathrm{~dB} / \mathrm{cm}$.

The quality of the inductance coil is determined by the loss-angle tangent or by the inverse value - the quality 
factor:

$\mathrm{Q}_{\mathrm{L}}=1 / \operatorname{tg} \delta_{\mathrm{L}}=\omega \mathrm{L} / \mathrm{R}_{\mathrm{L}}$, and the capacitor quality factor is

$\mathrm{Q}_{\mathrm{c}}=1 / \operatorname{tg} \delta_{\mathrm{c}}=1 / \omega \cdot \mathrm{C} \cdot \mathrm{R}_{\mathrm{c}}$

It follows that the quality factors are equal to the ratio of the reactance to the active resistance of the corresponding two-terminal circuit.

It is known that the modulus of the Poynting vector is defined as follows:

$\Pi=E^{2} / 120 \pi=E_{m}^{2} / 240 \pi \quad[4]$.

The power flow density of the electromagnetic radiation at distance $r$ from the source of radiation at a power of radiation flow $\mathrm{R}_{\text {total }}$ will be as follows:

$\Pi=\mathrm{P}_{\text {total }} / 4 \cdot \pi \cdot \mathrm{r}^{2}$.

For example, inside the designed resonator chamber with a diameter of $16 \mathrm{~cm}$ at distance $\mathrm{r}=10 \mathrm{~cm}$, the average power flow density will be as follows:

$\Pi=800 / 4 \cdot \pi \cdot 10^{2}=0,64 \mathrm{~W} / \mathrm{cm}^{2}$.

Generally, the density of the flow of the electromagnetic radiation inside the designed resonator chamber will be in the range from 0.5 to $60 \mathrm{~W} / \mathrm{cm}^{2}$. Outside the chamber the intensity of the flow will be between 60 and $62 \mathrm{~W}(7 \ldots 7.8 \%$ of $800 \mathrm{~W}$ ). From the above, it follows that the density of the flow outside the perforated resonator chamber, namely at a distance of $10 \mathrm{~cm}$, is on the average equal to:

$\Pi=62 / 4 \cdot \pi \cdot 10^{2}=0,05 \mathrm{~W} / \mathrm{cm}^{2}$.

Permissible parameters of the power flow density for the equipment maintenance personnel at a distance of $1.5 \mathrm{~m}$ from superhigh frequency plants are $0.001 \mathrm{~W} / \mathrm{cm}^{2}$.

\section{Discussions}

For the first time the resonator cavities were theoretically and practically studied by M.S. Neyman, V.I. Bunimovich, G.V. Kisunko, N.N. Krylov and others.

Research results of Atabekov G.I., Bredikhin S.A., Borodin I.F., Bunimovich V.I., Bashilov A.M., Vorobyov V.A., Vyshemirsky F.A., Ginzburg A.S., Kudryavtsev I.F., Zolotin V.P., Kavetsky G.D., Krus G.N., Krylov N.N., Kisunko G.V., Neyman M.S., Lykov A.V., Novikova G.V., Nikolaev N.S., Pchelnikov Yu.N., Plaksin Yu.M., Rogov I.A., Tsuglenok N.V., Strebkov D.S., et al. show that the use of the energy of the electromagnetic radiation for processing of agricultural products is effective, as well as in heat treatment of raw milk.

\section{Conclusions}

As a results of the analysis of dielectric parameters of the studied fermented milk products, we have developed operational and technological schemes of product processing that provide multiple sequential effect of two types of heat: exogenous and endogenous. Innovative plants were developed for the implementation of operational and technological schemes of heat treatment: superhigh frequency-induction plant for heat treatment of curdy raw material and superhigh frequency butter melter, which have a technical novelty. Superhigh frequency-induction plant includes a rotating dielectric disc with through holes arranged radially to transport dielectric containers with raw material through the process chamber which is formed by two resonator cavities and induction heaters. The slots in the resonator cavities are made to transport containers with raw material and are attached to the evanescent-mode waveguide. A table made of ferromagnetic material is mounted under the disc, as the secondary winding of the inductor. The inductors (primary windings) are mounted below it in a circumferential direction. There is a window in the shielding cylindrical body to load and unload the product. Superhigh frequency butter melter comprises the perforated resonator chambers with generator units mounted outside so that the radiators are have been directed to the corresponding resonating chambers. A centrifugal pump connected to the pipeline is for the product pumping. Performance of superhigh frequency plant depends on the number of generator units with the resonator chambers and their power.

The parameters of the process chamber with superhigh frequency power supply are justified: the electrodynamic system provides the implementation of temperature modes in the resonator cavity and the required power, as well as the structure of the electromagnetic field.

We have studied the distribution pattern of the electric field in superhigh frequency-induction plant and determined the energy deceleration coefficient depending on the diameter of the diaphragms in the waveguide. We have also justified the size of the slots in the chamber and the configuration of the developed evanescent-mode diaphragmed waveguide.

We have justified the process of induction heating, structural and technological parameters and modes of operation of superhigh frequency-induction plant.

The process chamber of superhigh frequency butter melter made of non-ferrimagnetic material for receipt of butter raw material and having perforated resonator chambers, provides screening of the flow of the electromagnetic radiation. The configuration of the chamber with a rectangular cross section is most convenient for installation of the main plant components, and the dimensions of the sides provide installation of two resonator chambers on the same horizontal plane, so that there is a distance between their bases of not less than a quarter wavelength. The height of the chamber is also matched by the number of the rows of the resonator chambers, affecting the performance of superhigh frequency plant. And the distance between the rows is also not less than a quarter wavelength, limiting the effect of the edge flow of the electromagnetic radiation of one generator on operation of the other magnetron.

Superhigh frequency resonator chambers of butter melter provide the continuous process of heat treatment of butter raw material due to the perforations. The dimensions of the perforations and their density on the surface of the chamber are matched with the edge flow of the electromagnetic radiation. And the lines of electric field are closed through raw material located on the outer surface of the chamber, which provides its preheating, and thus the reduction in viscosity. Raw material flows into the perforated chamber as 


\section{References}

1. Alyoshichev S.E. Hardware and technological analysis of the process of butter production as applied to the tasks of humidity stabilization / S.E. Alyoshichev // Dep. in VINITI. "The problems of equipment and technology of food production. SPb GUNiPT 2005. - pp. 156...159.

2. Alekushin A.E. How to choose equipment for butter production / A.E. Alekushin, V.M. Kuznetsov // Food Industry. - 2005. - No. 8. pp. $62 \ldots 63$

3. Alabovsky A.N. Technical thermodynamics and heat transfer / A.N. Alabovsky, I.A. Neduzhy. - Kiev: High School, 1990. - 255 p.

4. Belotserkovsky G.B. Fundamentals of radio engineering and antennas / G.B. Belotserkovsky. - M.: Soviet Radio, 1979. - Part 1. Fundamentals of radio engineering. $-368 \mathrm{p}$.

5. Askin I.M. Calculation of electromagnetic fields / I.M. Askin - M.: Energoizdat, 1959. - 385 p.

6. Azarov B.M. Technological equipment of food production / B.M. Azarov. - M.: Agropromizdat, 1988. - 463 p.

7. Atabekov G.I. Theoretical foundations of electrical engineering / G.I. Atabekov, S.D. Kupalyan, A.B. Timofeev, S.S. Khukhrikov. - M. L. Energiya, 1966. -280 p.

8. Naumenko O.V. Superhigh frequency-induction plant with the diaphragmed waveguide / O.V. Naumenko // International scientifictheoretical and applied journal Bulletin of the Chuvash State Pedagogical University n.a. I.Ya. Yakovlev. - Cheboksary: ChSPU, 2013, No. 4-2 (80), pp. 133-136. ISSN 1680-1709

9. Naumenko O.V. Development and substantiation of the parameters of superhigh frequency plants for processing of fermented milk products / O.V. Naumenko, G.A. Alexandrov, D.P. Kiryanov, G.V. Novikova // Publishing House "Pegas" LLC, Cheboksary, 2015, 106 p. Circulation 500 copies. ISSN 978-5-91225-069-9.

10. Fedyanovich V.I. Averaged boundary conditions for the band grids with losses. // Radio engineering and electronics. Vol. 37, No. 7. M.: RAS, 1992. - pp. 1180...1183.

11. Sviridov V.T., Superhigh frequency electronics / V.T. Sviridov, Yu.N. Pchelnikov - M.: Radio and communications, 1981. - 96 p. 